\title{
A Cyanobacterial Circadian Clock Based on the Kai Oscillator
}

\author{
T. KONDO \\ Division of Biological Science, Graduate School of Science, Nagoya University and SORST/CREST, \\ Japan Science and Technology Agency, Furo-cho, Chikusa-ku, Nagoya 464-8602, Japan
}

\begin{abstract}
In the cyanobacterium Synechococcus elongatus PCC 7942, the products of three genes (kaiA, kaiB, and kaiC) have been identified as essential components of the circadian clock. Recently, we reconstituted the self-sustainable circadian oscillation of the KaiC phosphorylation state by incubating purified $\mathrm{KaiC}$ with $\mathrm{KaiA}, \mathrm{KaiB}$, and ATP. This in vitro oscillation persisted for at least three cycles and the period was compensated against temperature changes. Period lengths observed in vivo in various $\mathrm{kaiC}$ mutants were consistent with those measured using in vitro mixtures containing the respective mutant KaiC proteins. These results demonstrate that the oscillation of KaiC phosphorylation is the primary pacemaker of the cyanobacterial circadian clock and reveal a novel function of proteins as timing devices that govern cellular metabolism. We further analyzed four aspects of the KaiC phosphorylation cycle in vitro: the interactions among $\mathrm{KaiA}, \mathrm{KaiB}$, and $\mathrm{KaiC}$; the functions of the two phosphorylation sites, the energetics that determine the circadian period, and the mechanisms that synchronize the components of the Kai oscillator. From these analyses, we have proposed a circadian program consisting of the three proteins that keeps biological time in a living cell.
\end{abstract}

\section{INTRODUCTION}

From bacteria and fungi to plants and animals, circadian clocks are ubiquitous endogenous biological timing mechanisms that adapt to daily alterations under environmental conditions. Cyanobacteria are the simplest organisms known to exhibit circadian rhythms. Thus, the cyanobacterium Synechococcus elongatus PCC 7942 (hereafter, Synechococcus) is an experimental model for the study of molecular mechanisms underlying these types of clocks (Kondo et al. 1993). Saturation mutagenesis revealed that three adjacent genes ( $k a i A, k a i B$, and $\mathrm{kaiC}$ ) and their protein products are essential for circadian rhythm generation. The levels of kaiBC mRNA and KaiC protein oscillate in a circadian manner, the latter of which is delayed by about 6 hours. Continuous overexpression of KaiC nullifies circadian rhythms through an amelioration of kaiBC expression, whereas a transient increase in KaiC sets the phase of the rhythm. These observations led to a model of the cyanobacterial transcription-translation-based oscillator (TTO) that explains prokaryotic circadian rhythm generation (Ishiura et al. 1998); in this model, KaiC and KaiA negatively and positively regulate $\mathrm{kaiBC}$ transcription, respectively.

This TTO model, however, did not explain how this loop obtains the characteristics unique to a circadian clock, i.e., circadian periodicity and stability against alterations in temperature and metabolic activity. A number of physiological studies have shown that these characteristics are essential for the oscillator to contribute to a fitness of organisms in their environment. Therefore, understanding the molecular basis of these features is an important goal in circadian biology. Our extensive screening for clock mutants in Synechococcus revealed that most of the period mutations were mapped to the kai gene cluster. In particular, some single-amino-acid substitutions in KaiC result in periods as short as 14 hours or as long as 60 hours (Fig. 1). To date, mutations in other genes of Synechococcus have only resulted in small changes in the circadian period, even if such mutation affects the amplitude of the rhythm severely. Therefore, KaiC is the primary determinant of the circadian period of cyanobacteria. One candidate for the molecular process that determines the circadian period is the phosphorylation of KaiC, which exhibits a robust circadian rhythm in its phosphorylation level in vivo (Iwasaki et al. 2002).

Although experimental evidence suggested that KaiC phosphorylation is an important contributor to circadian oscillation, it was not clear how this phosphorylation mediated circadian rhythm generation. A breakthrough was obtained when we examined the phosphorylation state of $\mathrm{KaiC}$ in prolonged darkness (Fig. 2a), which showed that the phosphorylation state of KaiC robustly oscillated in the cell with a 24-hour period, even under conditions in which neither transcription nor translation of kaiBC was permitted (Tomita et al. 2005). This finding raised doubts about the TTO model in cyanobacteria. The fact that this oscillation exhibited stability against temperature and metabolic activity changes suggested that the pacemaker of the cyanobacterial circadian system was not a transcription-translation feedback loop but was instead the KaiC phosphorylation cycle itself. We then attempted to reconstitute an oscillating cycle of KaiC phosphorylation in vitro. Incubating $\mathrm{KaiC}$ with $\mathrm{KaiA}, \mathrm{KaiB}$, and ATP produced a self-sustainable circadian oscillation of KaiC phosphorylation (Fig. 2b) (Nakajima et al. 2005). This in vitro oscillation of KaiC phosphorylation persisted for at least three cycles and the period exhibited temperature compensation. Furthermore, changes in the circadian rhythm period length observed in vivo in various $\mathrm{kaiC}$ mutant strains were consistent with those measured in vitro when the incubations were performed with the respective mutant KaiC proteins (Fig. 2c). Therefore, the oscillation of KaiC phosphorylation was identified as the primary pacemaker of the cyanobacterial circadian clock.

How do the three Kai proteins interact to produce a pre- 


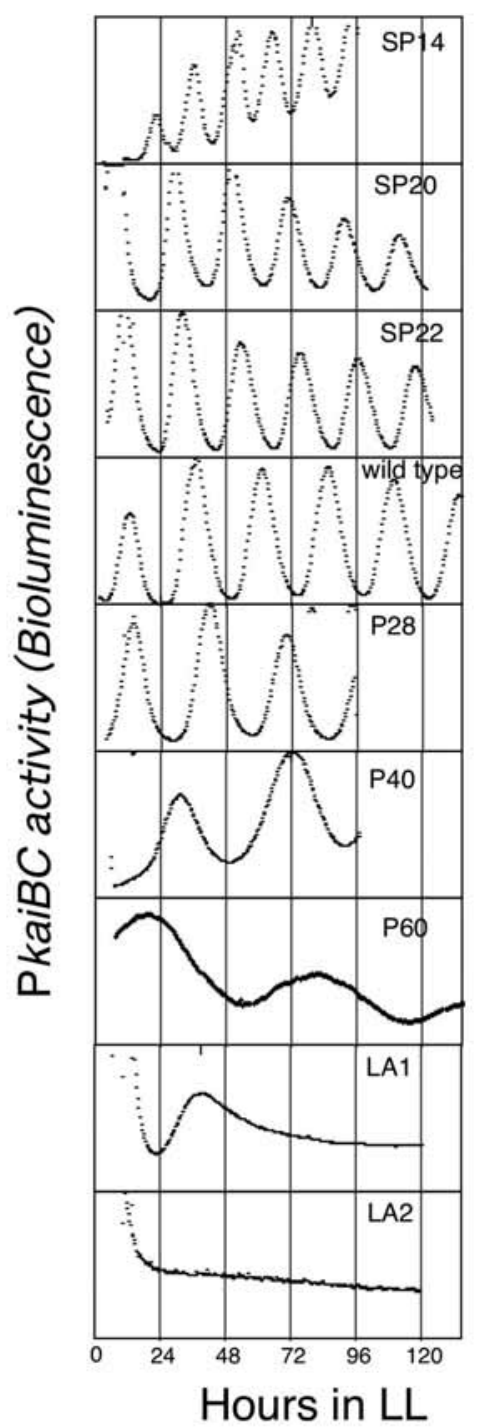

Figure 1. Period mutants of KaiC. Bioluminescence rhythm was examined using a bacterial luciferase reporter fused to the kaiBC promoter of period mutants of KaiC. All of the mutations were mapped to the $\mathrm{kaiC}$-coding region and resulted in single-aminoacid substitutions. For the loci and details of the mutant phenotypes, see Ishiura et al. (1998).

cise timing apparatus in the test tube? This chapter summarizes our recent studies about the mechanism underlying the KaiC phosphorylation cycle. Note also that this is a previously unrecognized phenomenon mediated by proteins, namely, proteins functioning as timing devices.

\section{KAI PROTEIN COMPLEX DYNAMICS IN THE KAIC PHOSPHORYLATION CYCLE IN VITRO}

Because our in vitro system is simple, we were able to quantitatively address the molecular dynamics of these three proteins. To elucidate how KaiC phosphorylation oscillation is regulated by interactions among the Kai proteins, we examined the associations of KaiA and KaiB with KaiC using immunoprecipitation analysis and gel-filtration chromatography (Kageyama et al. 2006). In this way, we

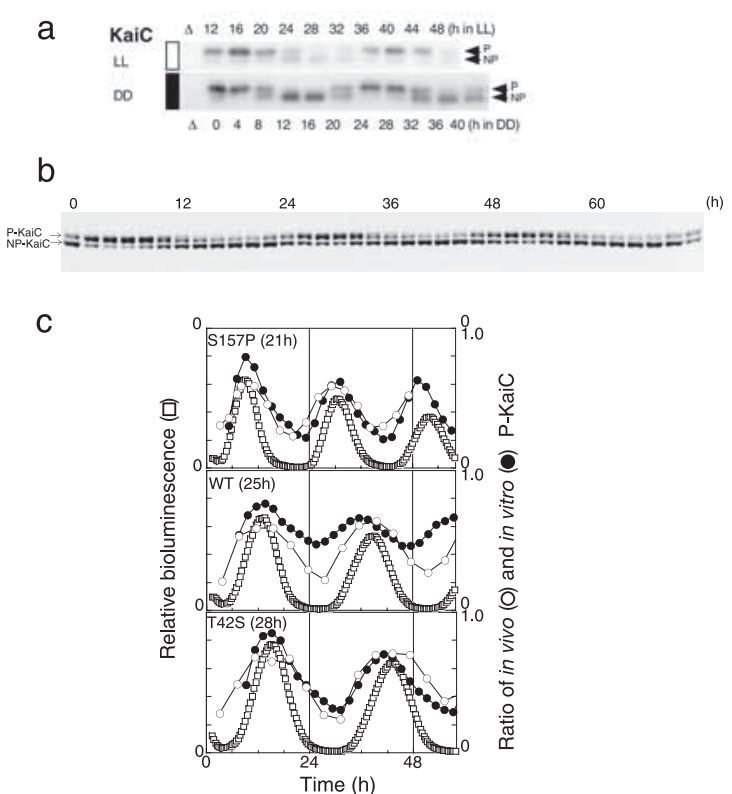

Figure 2. KaiC phosphorylation rhythms in vivo and in vitro. (a) Profiles of KaiC expression under constant light (LL) or constant dark (DD) conditions. The level of KaiC was estimated using the density of the signal on western blots of cell extracts taken at various time points under LL or DD conditions. The upper band in each lane represents phosphorylated $\mathrm{KaiC}$, whereas the lower band is dephosphorylated KaiC (Tomita et al. 2005). (b) KaiC phosphorylation rhythm in vitro. The three Kai proteins were synthesized in Escherichia coli, purified by chromatography, and mixed with $1 \mathrm{mM}$ ATP. Every 2 hours, aliquots were taken, subjected to SDSPAGE, and detected with Coomassie brilliant blue (CBB). (Upper band) Phosphorylated KaiC; (lower band) dephosphorylated KaiC. (c) KaiC phosphorylation rhythms in KaiC mutants. In vivo bioluminescence profiles and the ratios of phosphorylated $\mathrm{KaiC}$ in vivo and in vitro are shown for wild-type KaiC, the S157P mutant, and the T42S mutant. The period estimated from the bioluminescence rhythms are also shown for each strain.

confirmed that the sequences of Kai protein interactions and KaiC phosphorylation in vitro are similar to those observed in vivo (Kageyama et al. 2003; Kitayama et al. 2003).

Quantitative assessment of the biochemical data revealed the following dynamic protein associations: More than $95 \%$ of KaiA associated with KaiC throughout the cycle, whereas only $20-30 \%$ of the KaiC hexamers were associated with KaiA at any point in time. Because the ratio of KaiA dimers to KaiC hexamers in the mixture was $1: 1$, threefold to fourfold more KaiA dimers participated in the interaction with KaiC. Thus, the association between KaiA and KaiC should not be considered to be a simple one-to-one interaction. On the other hand, approximately $80 \%$ of the KaiC subunits were phosphorylated and dephosphorylated at the peaks and troughs of the cycle, respectively. If a stable association with KaiA is responsible for the phosphorylation and dephosphorylation of $\mathrm{KaiC}$, this range of KaiC phosphorylation in the cycle did not agree with the results. To solve this difficulty, we propose that KaiA dimers associate with KaiC hexamers without forming stable complexes. Instead, KaiA dimers repeatedly associate with and dissociate from KaiC hexamers, as if jumping from one KaiC to another (Fig. 3). Through this repeated action, KaiA could gradu- 


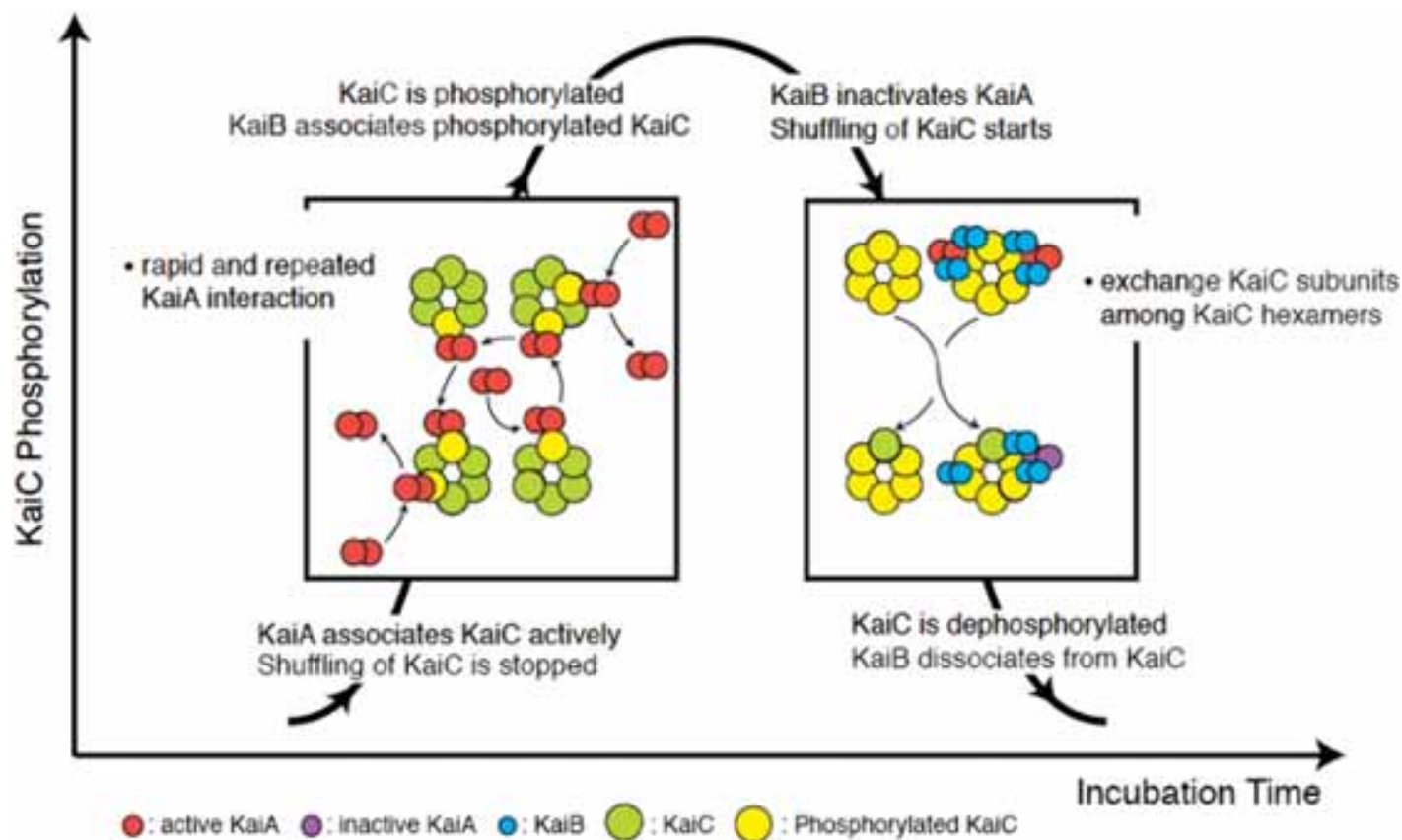

Figure 3. Associations between the three Kai proteins in an in-vitro-reconstituted system. The KaiC phosphorylation cycle is schematically illustrated on a plot of the incubation time versus KaiC phosphorylation. (Left frame) Principal protein dynamics for phosphorylation; (right frame) principal protein dynamics for dephosphorylation. See text for a more detailed explanation.

ally phosphorylate the KaiC subunits in the hexamers, resulting in $80 \%$ of the subunits being phosphorylated.

Increased phosphorylation of the KaiC hexamer could result in changes in the KaiC hexamer state, possibly through the addition of the charged phosphate groups. These changes could result in an increased affinity of KaiB for KaiC. In fact, KaiB tended to associate with the highly phosphorylated KaiC hexamers, forming the uniform complexes observed using gel-filtration chromatography. KaiC-bound $\mathrm{KaiB}$ traps and inactivates KaiA, promoting KaiC dephosphorylation. During the dephosphorylation phase, $60 \%$ or more of the KaiC hexamers remained free, whereas the remaining $40 \%$ associated with KaiA and KaiB. With the exception of the KaiAKaiC complexes, phosphorylated KaiC hexamers in any complexes are likely to be dephosphorylated, as are the free KaiC hexamers. After decreasing the degree of KaiC hexamer phosphorylation, KaiB dissociates from the hexamer and liberates KaiA, which then participates in the next round of KaiC phosphorylation (Fig. 3).

In the presence of ATP, KaiC forms a hexamer (Hayashi et al. 2003). We examined the potential for KaiC monomer exchange between two KaiC hexamers using FLAGtagged KaiC. We trapped KaiC hexamers containing KaiC-FLAG at various time points after the components of the oscillator were mixed. If KaiC monomers shuffled among other hexamers, a greater number of KaiC hexamers (up to twofold) would be pulled down using anti-FLAG antibodies. Actually, twice as much $\mathrm{KaiC}$ was precipitated following incubations for 4 hours or longer. This result clearly indicates that the KaiC monomers were shuffled between the hexamers in a manner that correlated with the temporal characteristics of the KaiC hexamers (Fig. 3). The shuffling of KaiC monomers may allow the dephos- phorylation to be equalized among KaiC hexamers with different configurations. We discuss this issue in a later section of this chapter. Similar results of Kai protein interaction and KaiC monomer exchange were also reported using different methods (Pattanayek et al. 2006). The shuffling of KaiC monomers between different phosphorylation states was also predicted to be important for the stability of the oscillator (Emberly and Wingreen 2006).

\section{A SEQUENTIAL PROGRAM OF KaIC PHOSPHORYLATION}

Previously, we identified two phosphorylation sites in KaiC at serine 431 (S431) and threonine 432 (T432), both of which are required for rhythm generation in vivo (Nishiwaki et al. 2004; Xu et al. 2004). To individually examine the phosphorylation states of S431 and T432, we employed nanoflow-liquid chromatography coupled with electrospray ionization-mass spectrometry and improved SDS-PAGE (Fig. 4a) (Nishiwaki et al. 2007). We found that the KaiC phosphorylation cycle was a sequence of four steps as shown in Figure 4b. To clarify the mechanisms underlying the sequential reactions, we mutated $\mathrm{KaiC}$ at the phosphorylation sites, introducing an alanine or glutamic acid (E)/aspartic acid (D) residue to mimic the dephosphorylated or phosphorylated states, respectively. Using these mutants, we found that the phosphorylation state of each residue regulated the phosphorylation/ dephosphorylation of the other residue. As illustrated in Figure $4 \mathrm{~b}$, the results suggested that the product of step 1 (T432 phosphorylation) allowed the subsequent reaction in step 2 (S431 phosphorylation) to proceed efficiently. Similarly, in steps 3 and 4, dephosphorylated T432 facilitated the dephosphorylation of S431, resulting in the 
a

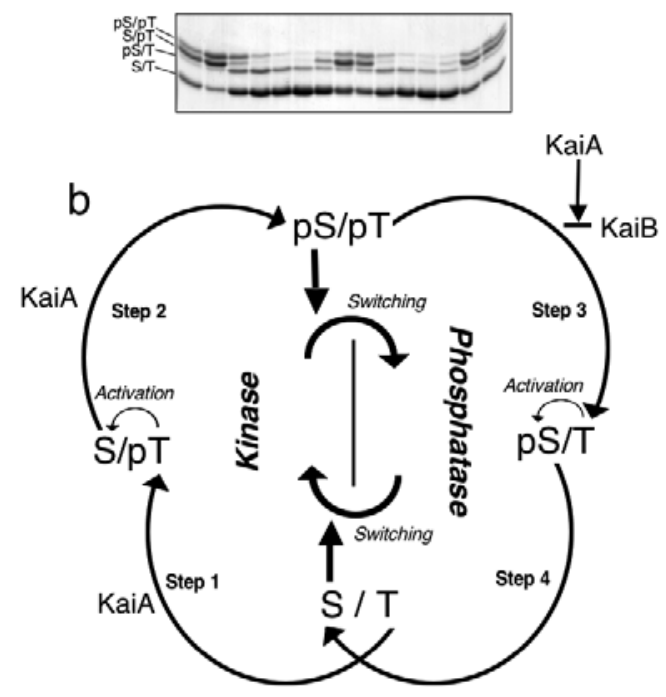

Figure 4. Phosphorylation profiles of the two phosphorylation sites of KaiC. (a) Aliquots of the reaction mixtures were collected at 4-hour intervals, mixed with $\left[\gamma_{-}{ }^{32} \mathrm{P}\right] \mathrm{ATP}$, and incubated for an additional 30 minutes. Samples were subjected to SDSPAGE. After CBB staining, four phosphorylated states of KaiC were detected. Phosphate was incorporated mainly into the two upper bands. (b) Model of the KaiC phosphorylation cycle. The KaiC phosphorylation cycle consists of four sequential reactions. During steps 1 and 2, KaiC acts as an autokinase, whereas during steps 3 and 4, KaiC functions as an autophosphatase. Phosphorylation of S431 switches KaiC from an autokinase to an autophosphatase, whereas dephosphorylation of S431 to form the completely dephosphorylated form $(\mathrm{S} / \mathrm{T})$ switches KaiC from an autophosphatase to an autokinase. Phosphorylation of T432 promotes the phosphorylation of S431, whereas dephosphorylation of S431 promotes dephosphorylation of T432.

completely dephosphorylated S/T form. Thus, once KaiA and ATP promote the phosphorylation of T432, the four steps of the cycle are programmed to proceed within an individual KaiC molecule.

Importantly, between steps 2 and 3, the phosphorylated S431 switches KaiC activity from an autokinase to an autophosphatase. In the same way, switching from the autophosphatase activity to the autokinase activity takes place between steps 4 and 1 with the dephosphorylation of S431. Note that in either step, phosphorylation/dephosphorylation of T432 precedes the change in the S431 phosphorylation state. This mechanism facilitates the in vitro oscillation of KaiC phosphorylation. It is also important to note that the dual phosphorylation mechanism would contribute to the hysteresis in the switching, which may be caused by allosteric changes in the structure of KaiC (Fig. 4b).

We found that KaiA and KaiB had little effect on the dephosphorylation of KaiC-DT (S at 431 was replaced with D) or KaiC-SE (T at 432 was replaced with E), suggesting that the switching of KaiC from an autokinase to an autophosphatase is predominantly regulated by the phosphorylation state of KaiC (step 3). Furthermore, our previous results from a radioactive phosphate uptake assay showed that a small amount of phosphate was incorporated into KaiC even when KaiA was not included in the reaction
(Iwasaki et al. 2002; Kitayama et al. 2003). We also confirmed that the ratio of phosphorylated serine and threonine was not altered by the presence of KaiA, whereas KaiA increased the overall level of phosphorylation at both residues (Iwasaki et al. 2002). Dephosphorylation of S431 (step 4) proceeded efficiently in the absence of KaiA and KaiB. Taken together, these observations indicate that KaiC potentially can progress through all of the phosphorylation cycle steps without KaiA and $\mathrm{KaiB}$. In other words, dual phosphorylation sites of KaiC make KaiC function as a "flywheel" for the phsophorylation cycle.

\section{THE ATPASE ACTIVITY OF KAIC SERVES AS THE BASIC TIMING MECHANISM OF THE CIRCADIAN CLOCK}

We then examined the molecular basis that defines the circadian period length. Because the circadian period length and its stability over a range of temperatures are essential for an adaptive circadian clock (Dunlap et al. 2004), the mechanism that defines the period length is a critical feature of a circadian oscillator. In contrast, a selfsustained oscillating loop could be more generally found for a number of processes in living cells.

KaiC, which has two ATP-binding motifs (Ishiura et al. 1998), was expected to display a robust ATPase activity because the hexameric structure of KaiC resembles that of a number of DNA-associated ATPases (Leipe et al. 2000). However, so far, KaiC only shows slow kinase and phosphatase activities. By examining ATP consumption during the KaiC phosphorylation cycle, we found that KaiC hydrolyzes 15-20 ATP molecules per cycle (Terauchi et al. 2007). Although the ATPase activity of KaiC was extremely low, this activity was essential for the phosphorylation rhythm to persist. To examine the ATPase activity precisely, we monitored ADP production in the reaction mixture (Fig. 5a). We found that the ATPase activity of KaiC oscillated with a circadian period when KaiA and KaiB were present in the mixture. Interestingly, even in the absence of KaiA and KaiB, the ATPase activity remained constant at a level that corresponded to the average level observed under rhythmic conditions (14.5 molecules day ${ }^{-1}=1.7 \times 10^{-4}$ molecules $\mathrm{s}^{-1}$ ). As was the case for the phosphorylation activity of KaiC (Iwasaki at al. 2002), KaiA stimulated the ATPase activity of KaiC and KaiB decreased the ATPase activity (Kitayama et al. 2003). We also found that a truncated variant of KaiC (KaiC-CI; residues 1-250 of KaiC), which lacked the phosphorylation sites, showed $70 \%$ of the basal activity, demonstrating that ATP hydrolysis was not entirely dependent on the kinase activity.

The thermal sensitivity of the activity was consistent with the thermal sensitivity of the cyanobacterial clock in vivo (Terauchi et al. 2007). As was done for the KaiC phosphorylation rhythm, we confirmed that the ATPase activity of $\mathrm{KaiC}$ in the presence of KaiA and $\mathrm{KaiB}$ was temperaturecompensated in the range from $25^{\circ} \mathrm{C}$ to $35^{\circ} \mathrm{C}$ (Fig. 5b). Surprisingly, the ATPase activity in KaiC-only incubations showed a considerably stronger level of temperature compensation; i.e., the activity was fairly constant at all temperatures examined with a $\mathrm{Q}_{10}$ coefficient of approximately 
a
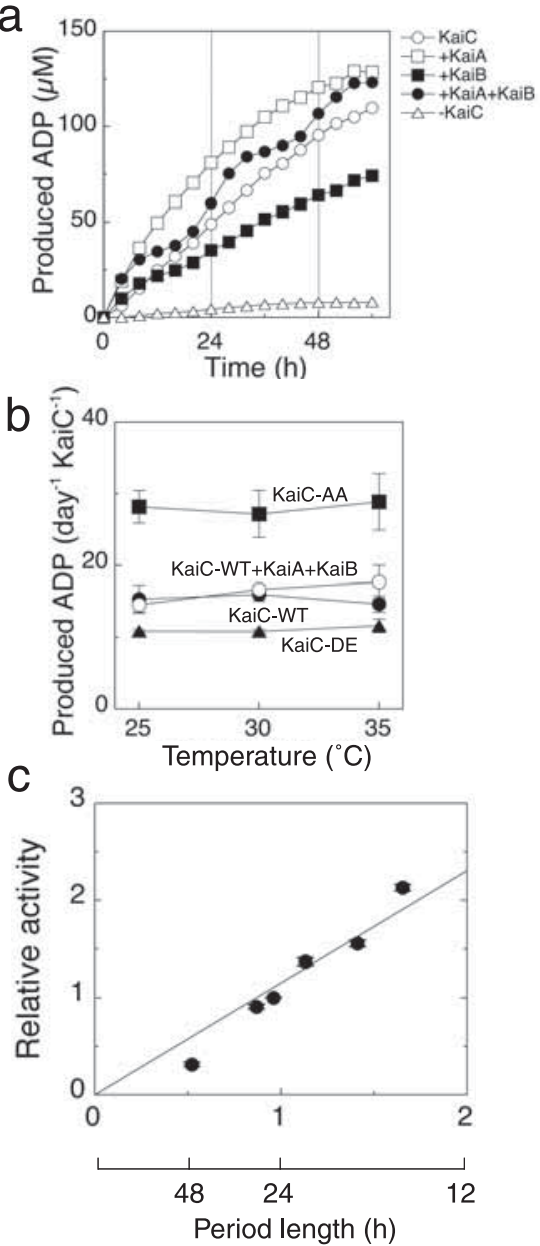

Figure 5. ATPase activity of KaiC. (a) KaiC-mediated ADP accumulation. KaiC was incubated under standard conditions with or without KaiA or KaiB at $30^{\circ} \mathrm{C}$. The ADP concentration in the reaction mixture was measured using high-performance liquid chromatography. The ADP level in a mixture containing only KaiA and KaiB (open triangles) is shown as a negative control. (b) Temperature compensation of the ATPase activity of KaiC. KaiC protein was incubated with 1 mM ATP at $25^{\circ} \mathrm{C}, 30^{\circ} \mathrm{C}$, or $35^{\circ} \mathrm{C}$ in the presence (open circles) or absence (closed circles) of KaiA and KaiB. KaiC-AA (squares) and KaiC-DE (triangles) were also examined under these conditions. (c) ATPase activity of KaiC and the circadian frequency. The ATPase activities of wild-type KaiC and five KaiC period mutant proteins (T42S, S157P, A251V, R393C, and F470Y) were measured in the absence of KaiA and KaiB. The ATPase activities of KaiC are plotted against the frequencies of the in vivo bioluminescence rhythms (reciprocal of the period length). The activity level observed for wild-type $\mathrm{KaiC}$ was set at 1.0.

1.0. This result indicated that the temperature compensation of the KaiC ATPase activity is an inherent property of the KaiC molecule. Furthermore, the ATPase activities of KaiC-AA (mimics dephosphorylated KaiC) and KaiC-DE (mimics phosphorylated KaiC) were also temperaturecompensated, whereas levels of ATPase activity were altered by these mutations. This result indicates that the temperature compensation of the ATPase activity is not dependent on the KaiC phosphorylation state.
We selected three mutants from more than $900 \mathrm{kaiC}$ mutants generated by polymerase chain reaction (PCR)based mutagenesis as "pure" period mutants. Each of these mutants had a bioluminescence profile that was identical to that of the wild-type strain, except for the period length. All other rhythmic phenotypes were almost identical to those of the wild-type strain (K. Imai et al., unpubl.). Using these mutants, we found that the period lengths of the expression rhythms of kaiBC in vivo were consistent with those of the respective in vitro KaiC phosphorylation rhythms (Nakajima et al. 2005). We measured the ATPase activities of the short-period mutant proteins (S157P and F470Y) and the long-period mutant and plotted the activities of the KaiC variants against the frequencies of the in vivo oscillations $\left(\right.$ period $^{-1}$ ). The plot clearly indicated that the frequencies were directly proportional to the ATPase activities (Fig. 5c), i.e., the basal ATPase activity of KaiC dictates the circadian period length of cyanobacteria: The higher the activity, the faster the clock ticks. In particular, the linear correlation indicates that the circadian pacemaker depends directly on the energy provided by ATP hydrolysis. In other words, the same amount of energy (hydrolysis of 15 ATP molecules per KaiC monomer) is required for one period of the circadian cycle. This finding represents the first description of a simple biochemical reaction acting as a circadian timekeeper.

ATPases interact with various partners to convert the energy of ATP hydrolysis into mechanical forces (Ye et al. 2004). Like other members of the RecA superfamily, KaiC contains a RecA-type nucleotide-binding domain, which converts the chemical energy of ATP into mechanical energy that is harnessed to move the protein along macromolecules. To the best of our knowledge, the activity of KaiC (15 ATPs day ${ }^{-1}$ ) is substantially lower than any other protein in this family; e.g., RuvB - a protein that is involved in DNA recombination-hydrolyzes $8 \times 10^{3}$ ATP day $^{-1}$ even in the absence of substrate DNA (Marrione and Cox 1995). To explain the extraordinarily weak and temperature-compensated activity of $\mathrm{KaiC}$, we hypothesize that the energy of ATP hydrolysis is not transferred to another molecule but is instead passed to KaiC itself, thereby lowering the observed activity (Fig. 6).

Similar to many RecA-like ATPases, KaiC forms a hexameric ring (Pattanayek et al. 2004); these RecA-like ATPases undergo conformational changes in response to ATP binding and hydrolysis (Wang 2004; Ye at al. 2004). Conformational changes in ATPases, such as p97 and $\mathrm{ClpB}$, have been observed during their ATPase cycles (Rouiller et al. 2002; Lee et al. 2007). Thus, after the formation of the hexamer structure, hydrolysis of ATP in KaiC may result in an immediate conformational change in the hexamer that decreases the ATPase activity to an uncommonly low level. Moreover, this autoregulation of the ATPase activity could be the basis for the temperature independence of the activity, because as the activity increases with increasing temperatures, the degree to which the activity is inhibited also increases. Analysis of the submolecular mechanisms underlying the ATPase reaction in the $\mathrm{KaiC}$ hexamer should provide valuable information regarding the molecular basis of the circadian period. In addition, the similarities with the stability of an 


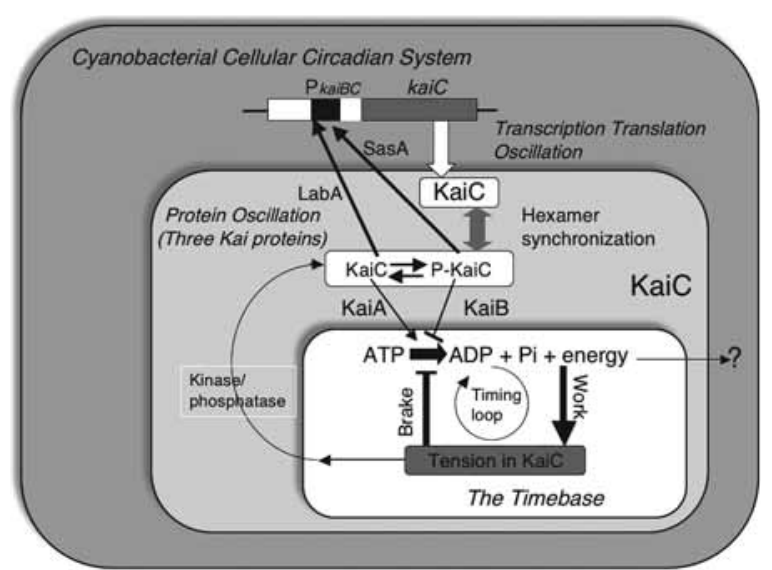

Figure 6. Cyanobacterial circadian system is defined by KaiC ATPase activity. A schematic diagram of the KaiC ATPase activity, the phosphorylation/dephosphorylation cycle, and the transcription-translation cycle. See text for a detailed explanation.

electric feedback amplifier should also be noted. When an amplifier forms a negative feedback loop that suppresses its native gain (open-loop gain) to $1 / 30$ or lower of the native value, the closed gain of the feedback circuit should become constant and insensitive to a drift in the open-loop gain. Assuming that the open-loop gain is the native ATPase activity of KaiC, which is much higher than the observed level, the extremely weak but temperature-compensated properties of the KaiC ATPase activity can be explained.

How is the ATPase activity of KaiC phenotypically expressed as the circadian period of the phosphorylation cycle? It is important to note that the ATP hydrolysis and phosphorylation activities of KaiC may influence each other (Fig. 6). In fact, the ATPase activity was influenced by KaiA, KaiB, and the phosphorylation state of KaiC. Moreover, these three factors may regulate the ATPase activity both negatively and positively, because the associations between these proteins and $\mathrm{KaiC}$ and the phosphorylation of KaiC are rhythmic (Kageyama et al. 2006). On the other hand, the ATPase activity may influence the kinase activity of KaiC, possibly by inducing a conformational change in the structure of either monomeric or hexameric KaiC, as shown by the finding that both activities of KaiC oscillate with the same phase angle. Therefore, these mutual couplings between the ATPase and kinase activities of KaiC may generate a self-sustained oscillation through two processes that resonate with a circadian period (Fig. 6). Note also that such coupling would be based on the duplicated structure of KaiC, which combines two activities together into a single protein molecule.

These couplings appear to conform to the principles of an electric back-coupled oscillator, in which alternating energy transfer between two components is partially coupled with a feedback circuit containing two components that periodically resonate with a time constant defined by the interaction between the two components. For example, energy that alternately accumulates as capacitance and inductance generates a damping oscillation that serves as the time base of a self-sustained oscillation when it is coupled with feedback circuits. Thus, the ATPase activity of KaiC and the accumulation of stress in the structure of KaiC could function as the time base of the phosphorylation rhythm when it is coupled with the kinase/phosphatase activity of KaiC through interactions with KaiA and KaiB. The transition of the conformational states during the ATPase cycle may strain the KaiC structure, which would be followed by relaxation of the tension, as was suggested to occur in the GroEL allosteric transitions (Hyeon et al. 2006).

\section{AUTONOMOUS SYNCHRONIZATION OF THE KAIC PHOSPHORYLATION RHYTHM}

In the in vitro reconsitution experiments, mixing three proteins with ATP should reset the oscillation. However, under continuous temperature conditions, the oscillation is expected to be a damped oscillation, because, apparently, there are no mechanisms to sustain the oscillation under our constant temperature conditions. We examined the KaiC phosphorylation cycle during prolonged incubations. As shown in Figure 7a, the KaiC phosphorylation rhythm persisted for 10 days without damping as long as the system was replenished with ATP. This suggests that the individual oscillating KaiC units were precisely synchronized in the in vitro system. We then tested the potential synchronization of the KaiC phosphorylation rhythm by mixing together samples with six different phases and then observing KaiC phosphorylation in the mixture (Fig. 7b). If KaiC phosphorylation in each sample oscillated independently, the overall phosphorylation ratio within the mixture should have averaged out to a constant value. We, however, found that almost immediately after mixing the samples, the mixture exhibited a phosphorylation rhythm with an amplitude that was comparable to those observed in the original individual samples. This observation suggests that the phosphorylation of the individual KaiC units was rapidly synchronized (Ito et al. 2007).

We then prepared two KaiC proteins that were labeled with different fluorescent tags that allowed us to trace their respective phosphorylation states. We combined two samples of the fluorescent KaiC proteins with different oscillatory phases and observed the phosphorylation of the labeled KaiC proteins. After the samples were mixed, we found that the KaiC sample that was originally in the dephosphorylation phase was dephosphorylated as was observed for the original sample. In contrast, the KaiC sample originally in the phosphorylation phase either was dephosphorylated or was inhibited from proceeding with the phosphorylation reaction. After the phosphorylation ratios in the two KaiC samples equalized, they began to be phosphorylated synchronously.

We previously demonstrated that KaiC monomers shuffle between the hexamers (Kageyama et al. 2006). When KaiC hexamers in the same phase were combined, pulldown assays with wild-type and FLAG-tagged KaiC demonstrated that monomer shuffling occurred only during a limited 4-hour period early in the dephosphorylation phase. Shuffling among KaiC hexamers in the early dephosphorylation phase would allow the phosphorylation states to synchronize, leading to the robust oscillation 


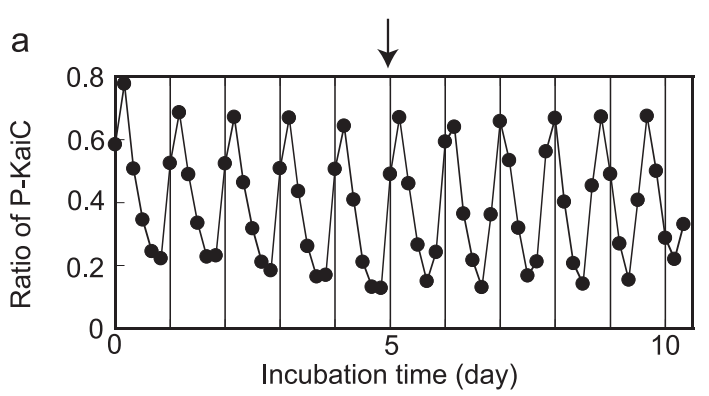

b
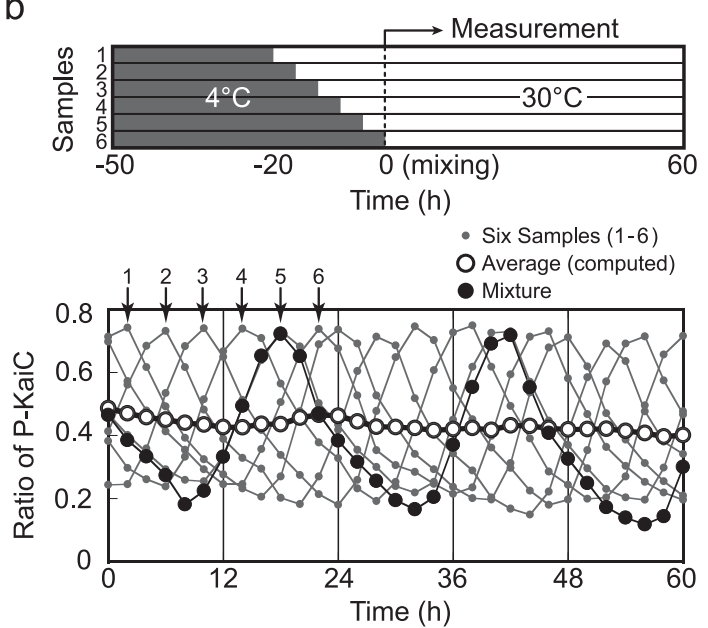

Figure 7. Synchronization of the in vitro KaiC oscillation. (a) Solution containing recombinant Kai proteins was incubated for 10 days under standard conditions. Five days after the incubation began, ATP was added to the solution to a final concentration of $1 \mathrm{~mm}$ (arrow). (b) After the standard sample was kept at $4^{\circ} \mathrm{C}$ for more than 30 hours, the temperature was raised to $30^{\circ} \mathrm{C}$ at various time points. At time 0 , aliquots of six samples (samples 1-6) with different phases were combined. (Gray dots) Phosphorylation ratios of the six samples; (black circles) phosphorylation ratios of the mixture. (Open circles) Arithmetic mean of the phosphorylation ratios from the six samples at each time point. The $x$ axis represents the period of time after mixing.

shown in Figure 7a. In short, the inevitable variance between the phosphorylation cycles of individual hexamers caused by thermal noise can be reset during each cycle. We also found that during the shuffling phase, KaiC monomers were able to shuffle between hexamers in different phases. This suggested that KaiC hexamers that contain a heterogeneous population of KaiC phosphorylation states are also likely to exchange monomers. Our model suggests that KaiC monomers within a hexamer in the phosphorylation phase switch to the dephosphorylation phase when monomers in the dephosphorylation phase are integrated into the hexamer. This process explains the results observed when KaiC proteins with different phosphorylation phases are mixed in vitro.

It is likely that the synchronization of the KaiC phosphorylation rhythm observed in vitro contributes to some extent to the robustness of the circadian rhythm in cyanobacterial cells. Because cells grown under constant light are able to divide more than twice a day (Kondo et al. 1997), each cell doubles the amount of Kai proteins due to de novo synthesis at least twice within one circadian cycle. Because newly synthesized KaiC protein is not phosphorylated, the KaiC phosphorylation rhythm in rapidly growing cells is constantly subjected to perturbations due to these new KaiC molecules. Although these perturbations would be expected to damp the KaiC oscillation, the amplitude and period of the phosphorylation cycle in rapidly dividing cells are similar to those in cells at a stationary phase or in cells under dark conditions. As observed in vitro, it is likely that newly synthesized KaiC proteins are subjected to an entraining process during the dephosphorylation phase, which results in a synchronization of the phosphorylation state and reaction direction at the beginning of next phosphorylation phase. Thus, the robustness of the circadian system in living cells may be principally achieved at the level of a chemical oscillator (Fig. 6).

Studies examining the circadian rhythms in single cells revealed that the circadian clock of individual cyanobacterial cells is extremely (Mihalcescu et al. 2004) robust when compared with other cellular oscillatory systems that are based on transcriptional networks. Oscillatory systems based on transcriptional regulatory networks are inevitably influenced by fluctuating concentrations of gene products due to the stochastic nature of transcriptional processes (Elowitz et al. 2002) and various external perturbations, such as cell division. The precision of mammalian circadian rhythms is thought to be achieved through cell-cell communication between oscillating cells. Although cyanobacterial cells do not communicate with each other with respect to circadian rhythm (Mihalcescu et al. 2004), communication (i.e., monomer shuffling) between the oscillating units (KaiC hexamers) renders the circadian oscillation of individual cells highly stable and precise. Such precision requires the participation of a relatively large number of oscillating units; indeed, an individual cyanobacterial cell contains approximately 10,000 KaiC proteins (Kitayama et al. 2003), a number that is comparable to the number of neurons in the mammalian suprachiasmatic nucleus.

\section{THE KAIC-BASED CELLULAR CIRCADIAN SYSTEM}

As shown above, the Kai-based chemical oscillator robustly functions in vitro, similar to a pendulum that is not connected to a downstream gear, allowing it to run without any external disturbances. On the other hand, the Kai-based oscillator in the cells is connected to a downstream process. Thus, the cellular circadian system involves transcription-translation processes; these processes supply the clock components that vary with the rate of cell proliferation. Even under such conditions, the circadian clock of the individual cell is extremely precise. To understand the intracellular dynamics of the KaiCbased circadian system, the following issues need to be examined: (1) the mechanism by which the Kai-based chemical oscillator drives circadian rhythms in genomewide transcription in cyanobacteria; (2) how rhythms in Kai protein synthesis and degradation as well as those in intracellular metabolic conditions are coupled with the Kai-based oscillator; and (3) how the Kai-based oscillator is entrained to day/night alterations in the environment. 
The linkage between KaiC and transcription is not mediated by a single pathway. One possibility is that KaiC activates gene expression via a two-component regulatory system, which includes the KaiC-binding histidine kinase SasA (Iwasaki et al. 2000). In sasAinactivated strains, kaiBC expression is markedly reduced, and circadian transcription rhythms are severely attenuated. Although SasA is not required for a basic oscillation, it may function as a connection between the KaiC phosphorylation cycle and transcription. To elucidate this pathway, we recently identified the DNA-binding protein RpaA as a cognate response regulator of SasA (Takai et al. 2006). Circadian transcription was severely attenuated in rpaA mutant cells, and the phosphotransfer activity from SasA to RpaA was dependent on the circadian state of the coexisting Kai protein complexes in vitro. Thus, we have proposed a model in which the SasARpaA two-component system mediates time signals from the enzymatic oscillator to drive genome-wide transcription rhythms in cyanobacteria.

The mechanism and significance of negative feedback regulation of $k a i B C$, however, have not been fully elucidated. We recently reported that the novel gene $l a b A$ is required for the negative feedback regulation by KaiC. Disruption of $l a b A$ abolished the transcriptional repression caused by overexpression of $\mathrm{KaiC}$ and elevated the trough levels of circadian gene expression, resulting in a low-amplitude phenotype (Taniguchi et al. 2007). In contrast, overexpression of labA significantly lowered the levels of circadian gene expression. Furthermore, genetic analysis indicated that $l a b A$ and $\operatorname{sas} A$ function in parallel pathways to regulate $k a i B C$ expression. These results suggest that the temporal information derived from the KaiCbased oscillator diverges into a LabA-dependent negative regulatory pathway and a SasA-dependent positive regulatory pathway. It is likely that quantitative information from KaiC is transmitted to RpaA through LabA, whereas SasA mediates the state of the KaiC-based oscillator. It should be noted that disruption of either pathway did not affect the circadian period as much as it affected the transcriptional levels of the downstream targets. These observations imply that both types of regulations are necessary for robust cellular rhythms, whereas the circadian pacemaker is dominated by the biochemistry of KaiC.

Another important characteristic of a circadian clock is entrainment of the circadian oscillators. We observed a synchronization of the circadian phosphorylation of the KaiC hexamers, which resulted from monomer shuffling. This mechanism contributes to the robustness of the circadian oscillation in the cell by entraining de-novo-synthesized KaiC to the previously established oscillation. A more dynamical assessment in cells based on the rhythmic synthesis and degradation of Kai proteins, however, would allow a quantitative understanding of the cyanobacterial oscillator. On the basis of classical observation of circadian rhythm, we have two models for the entrainment of the circadian clock. An evaluation of the intracellular dynamics of Kai protein synthesis, degradation, and cellular localization would address the nonparametric model. In addition, note that temperature would be an effective factor to entrain the circadian oscillation of cyanobacteria. Mori et al. (2007) reported phase shifting due to temperature shifts, whereas we found that in vitro KaiC phosphorylation rhythms can be entrained to a temperature cycle (T. Yoshida et al., unpubl.). On the other hand, changes in intracellular factors, such as the ATP level, the ratio of KaiA to $\mathrm{KaiC}$, $\mathrm{pH}$, and the concentrations of other metabolites produced by photosynthesis, can be used to test the parametric entrainment model; the effect of these parameters on the ATPase activity of KaiC would be the basis of this entrainment model.

\section{CONCLUSIONS}

After identifying the KaiC phosphorylation rhythm in vitro, we have elucidated the dynamics and mechanism of this autonomous oscillating system with biochemical and functional approaches. We found that the interactions between the three proteins are coupled with the phosphorylation program that is installed in the two phosphorylation sites of KaiC like a molecular flywheel. These unique intermolecular and intramolecular mechanisms are critical for the Kai protein oscillator of the cyanobacterial circadian clock.

Moreover, we found that the circadian period length and its stability are derived from the ATPase activity of KaiC. Note that our findings imply that the circadian period length, which should be a product of natural selection, is based on ATP hydrolysis, rather than the delicate balance of the time delay of clock gene expression. This strategy of an oscillator as an accurate time base is similar to what is observed in many electronic or physical oscillators. In these systems, mutually interacting components that can transform energy into different forms (e.g., inductance and capacitance or tension and inertia) define the time base of a self-sustaining oscillator network.

Our results reviewed here provide a basis for studying the physical machinery of this unique protein-based timer. Associations between the three Kai proteins, phosphorylation of $\mathrm{KaiC}$, and the KaiC ATPase activity may all be governed by intramolecular distortions of the KaiC structure. Fortunately, the atomic structures of the three Kai proteins have already been examined using X-ray crystallography and nuclear magnetic resonance (NMR) analyses (Golden et al. 2007). An understanding of KaiC at the atomic level, however, requires precise information regarding how the structure is modified with respect to KaiC functions. Thus, several snapshots of these configurations are crucial to allow the time profiles of the structural changes to be elucidated. It is important that these structural analyses be accompanied by an examination of their functional significance. Fortunately, more than 1000 KaiC mutants with previously characterized phenotypes are available for these types of analyses. It should be noted that the identification of $\mathrm{KaiC}$ as a time base is a novel function of proteins in general.

The dynamics of the Kai oscillator in the cell should also be examined for a complete understanding of the circadian system in these cells (see Fig. 6). We have shown that Kai oscillators are able to synchronize with each other due to monomer exchange between KaiC hexamers. The Kai oscillator, however, is subjected to various fluc- 
tuations in the rate of KaiC turnover. In a cell, KaiC turnover is subjected to negative feedback regulation, and the roles of KaiC in gene expression are not simply mediated by its phosphorylation status. Further studies of the feedback regulation as well as the integrated dynamics of the Kai oscillator and intracellular metabolism will elucidate mechanisms underlying the robustness of the cellular clock and its entrainment to day/night alterations.

\section{ACKNOWLEDGMENTS}

I thank the current and former lab members who contributed to our studies of the cyanobacterial circadian clock. We also thank Synechococcus elongates, which has developed a unique process to cope with the 24-hour periodicity observed on this planet. This research was supported in part a grant-in-aid from the Ministry of Education, Culture, Sports, Science and Technology of Japan (15GS0308 to T.K.).

\section{REFERENCES}

Dunlap J.C., Loros J.J., and DeCoursey P.J. 2004. Chronobiology: Biological timekeeping. Sinauer, Sunderland, Massachusetts.

Elowitz M.B., Levine A.J., Siggia E.D., and Swain P.S. 2002. Stochastic gene expression in a single cell. Science 297: 1183.

Emberly E. and Wingreen S.N. 2006. Hourglass model for a protein-based circadian oscillator. Phys. Rev. Lett. 96: 038303.

Golden S.S., Cassone V.M., and LiWang A. 2007. Shifting nanoscopic clock gears. Nat. Struct. Mol. Biol. 14: 362.

Hayashi F., Suzuki H., Iwase R., Uzumaki T., Miyake A., Shen J.R., Imada K., Furukawa Y., Yonekura K., Namba K., and Ishiura M. 2003. ATP-induced hexameric ring structure of the cyanobacterial circadian clock protein KaiC. Genes Cells 8: 287.

Hyeon C., Lorimer G.H., and Thirumalai D. 2006. Dynamics of allosteric transitions in GroEL Proc. Natl. Acad. Sci. 103: 18939.

Ishiura M., Kutsuna S., Aoki S., Iwasaki H., Andersson C.R., Tanabe A., Golden S.S., Johnson C.H., and Kondo T. 1998. Expression of a gene cluster kaiABC as a circadian feedback process in cyanobacteria. Science 281: 1519.

Ito H., Kageyama H., Mutsuda M., Nakajima M., Oyama T., and Kondo T. 2007. Origin of the resilience of the cyanobacterial circadian clock. Nat. Struct. Mol. Biol. (in press).

Iwasaki H., Nishiwaki T., Kitayama Y., Nakajima M., and Kondo T. 2002. KaiA-stimulated KaiC phosphorylation in circadian timing loops in cyanobacteria. Proc. Natl. Acad. Sci. 99: 15788 .

Iwasaki H., Williams S.B., Kitayama Y., Ishiura M., Golden S.S., and Kondo T. 2000. A KaiC-interacting sensory histidine kinase, SasA, necessary to sustain robust circadian oscillation in cyanobacteria. Cell 101: 223.

Kageyama H., Kondo T., and Iwasaki H. 2003. Circadian formation of clock protein complexes by KaiA, KaiB, KaiC and SasA in cyanobacteria J. Biol. Chem. 278: 2388.

Kageyama H., Nishiwaki T., Nakajima M., Iwasaki H., Oyama T., and Kondo T. 2006. Cyanobacterial circadian pacemaker: Kai protein complex dynamics in the KaiC phosphorylation cycle in vitro. Mol. Cell 23: 161.

Kitayama Y., Iwasaki H., Nishiwaki T., and Kondo T. 2003. $\mathrm{KaiB}$ functions as an attenuator of KaiC phosphorylation in the cyanobacterial circadian clock system. EMBOJ. 22: 2127.

Kondo T., Mori T., Lebedeva N.V., Aoki S., Ishiura M., and Golden S.S. 1997. Circadian rhythms in rapidly dividing cyanobacteria. Science 275: 224.

Kondo T., Strayer C.A., Kulkarni R.D., Taylor W., Ishiura M., Golden S.S., and Johnson C.H. 1993. Circadian rhythms in prokaryotes luciferase as a reporter of circadian gene expression in cyanobacteria. Proc. Natl. Acad. Sci. 90: 5672.

Lee S., Choi J.-M., and Tsai F.T.F. 2007. Visualizing the ATPase cycle in a protein disaggregating machine: Structural basis for substrate binding by ClpB. Mol. Cell 25: 261.

Leipe D.D., Aravind L., Grishin N.V., and Koonin E.V. 2000. The bacterial replicative helicase DnaB evolved from a RecA duplication Genome Res. 10: 5.

Marrione P.E. and Cox M.M. 1995. RuvB protein-mediated ATP hydrolysis: Functional asymmetry in the RuvB hexamer Biochemistry 34: 9809.

Mihalcescu I., Hsing W., and Leibler S. 2004. Resilient circadian oscillator revealed in individual cyanobacteria. Nature 430: 81.

Mori T., Williams D.R., Byrne M.O., Qin X., Egli M., Mchaourab H.S., Stewart P.L., and Johnson C.H. 2007. Elucidating the ticking of an in vitro circadian clockwork. PLoS Biol. 5: e93.

Nakajima M., Imai K., Ito H., Nishiwaki T., Murayama Y., Iwasaki H., Oyama T., and Kondo T. 2005. Reconstitution of circadian oscillation of cyanobacterial KaiC phosphorylation in vitro. Science 308: 414.

Nishiwaki T., Satomi Y., Kitayama Y., Terauchi K., Kiyohara R., Takao T., and Kondo T. 2007. A sequential program of dual phosphorylation of KaiC as a basis for circadian rhythm in cyanobacteria. EMBO J. 26: 4029.

Nishiwaki T., Satomi Y., Nakajima M., Lee C., Kiyohara R., Kageyama H., Kitayama Y., Temamoto M., Yamaguchi A., Hijikata A., Go M., Iwasaki H., Takao T., and Kondo T. 2004. Role of KaiC phosphorylation in the circadian clock system of Synechococcus elongatus PCC 7942. Proc. Natl. Acad. Sci. 101: 13927.

Pattanayek R., Wang J., Mori T., Xu Y., Johnson C.H., and Egli M. 2004. Visualizing a circadian clock protein: Crystal structure of KaiC and functional insights. Mol. Cell 15: 375.

Pattanayek R., Williams D.R., Pattanayek S., Xu Y., Mori T., Johnson C.H., Stewart P.L., and Egli M. 2006. Analysis of KaiA-KaiC protein interactions in the cyano-bacterial circadian clock using hybrid structural methods. EMBO J. 25: 2017.

Rouiller I., DeLaBarre B., May A.P., Weis W.I., Brunger A.T., Milligan R.A., and Wilson-Kubalek E.M. 2002. Conformational changes of the multifunction p97 AAA ATPase during its ATPase cycle. Nat. Struct. Biol. 12: 950.

Takai N., Nakajima M., Oyama T., Kito R., Sugita C., Sugita M., Kondo T., and Iwasaki H. 2006. A KaiC-associating SasARpaA two-component regulatory system as a major circadian timing mediator in cyanobacteria. Proc. Natl. Acad. Sci. 103: 12109.

Taniguchi Y., Katayama M., Ito R., Takail N., Kondo T., and Oyama T. 2007. labA: A novel gene required for negative feedback regulation of the cyanobacterial circadian clock protein KaiC. Genes Dev. 21: 60.

Terauchi K., Kitayama Y., Nishiwaki T., Miwa K., Murayama Y., Oyama T., and Kondo T. 2007. The ATPase activity of $\mathrm{KaiC}$ determines the basic timing for circadian clock of cyanobacteria. Proc. Natl. Acad. Sci. (in press).

Tomita J., Nakajima M., Kondo T., and Iwasaki H. 2005. No transcription-translation feedback in circadian rhythm of KaiC phosphorylation. Science 307: 251.

Wang J. 2004. Nucleotide-dependent domain motions within rings of the RecA/AAA $(+)$ superfamily. J. Struct. Biol. 148: 259.

Xu Y., Mori T., Pattanayek R., Pattanayek S., Egli M., and Johnson C.H. 2004. Identification of key phosphorylation sites in the circadian clock protein KaiC by crystallographic and mutagenetic analyses. Proc. Natl. Acad. Sci. 101: 13933.

Ye J., Osborne A.R., Groll M., and Rapoport T.A. 2004. RecAlike motor ATPases-Lessons from structures Biochim. Biophys. Acta 1659: 1. 


\section{$8_{\text {SYMPOS }}^{\infty}$ Cold Spring Harbor Symposia SYMPOSIA on Quantitative Biology}

\section{A Cyanobacterial Circadian Clock Based on the Kai Oscillator}

T. Kondo

Cold Spring Harb Symp Quant Biol 2007 72: 47-55

Access the most recent version at doi:10.1101/sqb.2007.72.029

References This article cites 30 articles, 15 of which can be accessed free at: http://symposium.cshlp.org/content/72/47.full.html\#ref-list-1

\section{License}

Email Alerting Receive free email alerts when new articles cite this article - sign up in the box at the Service top right corner of the article or click here.

To subscribe to Cold Spring Harbor Symposia on Quantitative Biology go to: http://symposium.cshlp.org/subscriptions 